\title{
Assessment of Response to Chemotherapy in Pancreatic Cancer with Liver Metastasis: CT Texture as a Predictive Biomarker
}

\author{
Sihang Cheng, Zhengyu Jin and Huadan Xue *
}

check for updates

Citation: Cheng, S.; Jin, Z.; Xue, H Assessment of Response to Chemotherapy in Pancreatic Cancer with Liver Metastasis: CT Texture as a Predictive Biomarker. Diagnostics 2021, 11, 2252. https://doi.org/ 10.3390/diagnostics11122252

Academic Editor: Damiano Caruso

Received: 25 October 2021

Accepted: 26 November 2021

Published: 1 December 2021

Publisher's Note: MDPI stays neutral with regard to jurisdictional claims in published maps and institutional affiliations.

Copyright: (C) 2021 by the authors. Licensee MDPI, Basel, Switzerland. This article is an open access article distributed under the terms and conditions of the Creative Commons Attribution (CC BY) license (https:/ / creativecommons.org/licenses/by/ $4.0 /)$.
Department of Radiology, Peking Union Medical College Hospital, Chinese Academy of Medical Sciences, Beijing 100730, China; chengsihangscu@foxmail.com (S.C.); zhengyu_jin@163.com (Z.J.)

* Correspondence: bjdanna95@hotmail.com; Tel.: +86-10-69159569

\begin{abstract}
In this paper, we assess changes in CT texture of metastatic liver lesions after treatment with chemotherapy in patients with pancreatic cancer and determine if texture parameters correlate with measured time to progression (TTP). This retrospective study included 110 patients with pancreatic cancer with liver metastasis, and mean, entropy, kurtosis, skewness, mean of positive pixels, and standard deviation (SD) values were extracted during texture analysis. Response assessment was also obtained by using RECIST 1.1, Choi and modified Choi criteria, respectively. The correlation of texture parameters and existing assessment criteria with TTP were evaluated using Kaplan-Meier and Cox regression analyses in the training cohort. Kaplan-Meier curves of the proportion of patients without disease progression were significantly different for several texture parameters, and were better than those for RECIST 1.1-, Choi-, and modified Choi-defined response ( $p<0.05$ vs. $p=0.398$, $p=0.142$, and $p=0.536$, respectively). Cox regression analysis showed that percentage change in SD was an independent predictor of TTP $(p=0.016)$ and confirmed in the validation cohort $(p=0.019)$. In conclusion, CT texture parameters have the potential to become predictive imaging biomarkers for response evaluation in pancreatic cancer with liver metastasis.
\end{abstract}

Keywords: pancreatic cancer; liver metastasis; chemotherapy; texture analysis; response

\section{Introduction}

Pancreatic cancer is among the top cancers with high mortality affecting over 200,000 deaths every year, worldwide [1,2]. Unfortunately, a large proportion of patients are identified at an advanced stage with poor prognosis, especially for metastatic pancreatic cancer, for which the liver is the most common site, accounting for $37-41.9 \%$ of the initially diagnosed cases, with a 5-year overall survival (OS) rate of $2 \%$, and a median life expectancy of less than 1 year [3,4]. FOLFIRINOX and gemcitabine-based chemotherapy regimens are common treatments for metastatic pancreatic cancer. It was observed that FOLFIRINOX was superior to gemcitabine alone in progression-free survival (PFS), response, and OS in the treatment of metastatic pancreatic cancer, and another phase 3 trial confirmed the superiority of gemcitabine plus nanoparticle albumin-bound paclitaxel (nab-paclitaxel) as well $[5,6]$. Assessment of treatment response to chemotherapy is important, especially for the non-responders, which might provide these patients opportunities to find more appropriate treatment plans in time.

The response evaluation criteria in solid tumors (RECIST) as a frequently used tool for the assessment of tumor response only takes size change of the lesions into account [7]. Choi et al. developed comprehensive criteria incorporating changes in both tumor size and attenuation, which defines partial response (PR) as a decrease of $>10 \%$ in tumor size or a decrease of $>15 \%$ in tumor attenuation. These criteria define progressive disease (PD) as a tumor size increase of $>10 \%$ without meeting the PR criteria [8]. The definition of PR according to the modified Choi criteria is a $15 \%$ reduction in enhancement and a $10 \%$ reduction in size [9]. No studies have been conducted to assess the response to chemotherapy in pancreatic cancer with liver metastasis using these criteria. 
Analysis of tumor lesion heterogeneity will reveal vital information concerning response to treatment $[10,11]$. CT texture analysis is an emerging technique used to process images and hence help to characterize lesion heterogeneity. This technique analyzes the relationship and distribution of pixel gray levels within a lesion and reveals spatial variations among individual gray patterns or levels $[10,12,13]$. Extracted features include kurtosis, skewness, mean of positive pixels (MPP), entropy, standard deviation (SD), and mean graylevel intensity (MI) [14]. It has been proved that CT texture analysis played an important role in the assessment of tumor response to various treatments and had the potential to establish a more precise assessment criteria for response evaluation [15-18]. However, texture analysis as a non-invasive tool for the assessment of chemotherapy treatment response in pancreatic cancer with liver metastasis has not been investigated before.

In this study, we assessed alterations in CT texture of metastatic liver lesions following gemcitabine-based chemotherapy in pancreatic cancer patients and then compared the effectiveness of this approach with existing assessment criteria in evaluating treatment response and determining time to progression (TTP).

\section{Materials and Methods}

\subsection{Study Population}

Prior to the study, approval of this study was waived by the institutional review board of Peking Union Medical College Hospital for its retrospective nature. Patients with pancreatic cancer with liver metastasis undergoing chemotherapy between September 2014 and October 2018 were identified from our institutional electronic medical database. Inclusion criteria: (a) They were pathologically diagnosed to be pancreatic cancer with liver metastasis; (b) they were chemotherapy naïve and received gemcitabine-based chemotherapy (gemcitabine plus an oral fluoropyrimidine anticancer agent, S-1) as first-line treatment; (c) baseline contrast-enhanced CT had been done in 2 weeks prior to treatment initiation; (d) contrast-enhanced CT had been done following treatment to monitor response. A total of 168 patients met the inclusion criteria. Patients were excluded if they had previously undergone radiofrequency or microwave ablation of the liver metastatic lesions $(n=17)$, had infiltrative HCC without any accurately delineable lesion $(n=20)$, died during the first 15 days after treatment initiation $(n=3)$, and were without a regular documented follow-up after chemotherapy initiation or until disease progression $(n=18)$.

\subsection{Follow-Up and Endpoints}

The follow-up (radiological, clinical, and biological) was carried out every 2 months as outlined by the institutional protocol. During follow-up, radiological assessment was performed with contrast-enhanced CT scans of the pelvis, abdomen, and thorax. TTP was defined as the time from the start of chemotherapy to radiologic progression, which was the chosen end point detailed by RECIST 1.1. Participants who did not exhibit radiologic progression at the end of the follow-up were censored.

\subsection{Protocol of CT Examination}

All images were acquired in the Department of Radiology at our hospital. All the scans were done on 128-detector CT scanners (Siemens SOMATOM Definition Flash, Siemens Healthcare, Forchheim, Germany). The scanning parameters were as follows: tube voltage, $120 \mathrm{kVp}$; tube current, $150 \mathrm{mAs}$ (thorax) or $200 \mathrm{mAs}$ (abdomen and pelvis) with dose modulation; gantry rotation time, $0.5 \mathrm{~s}$; table increment $46.8 \mathrm{~mm}$ per rotation; matrix $512 \times 512$. Images were routinely reconstructed with $5.0 \mathrm{~mm}$ slice thickness and $5.0 \mathrm{~mm}$ intervals. Non-ionic contrast media (Ultravist, $370 \mathrm{mg}$ of iodine per milliliter, Schering, Berlin, Germany) were injected with $1.5 \mathrm{~mL}$ per kilogram of body weight at a rate of $3.0 \mathrm{~mL} / \mathrm{s}$ using an automatic power injector. Bolus tracking was applied, and the thorax and upper abdomen (from the supraclavicular fossa to the iliac crest) were imaged in the arterial phase of enhancement (25-s delay), and the abdomen and pelvis (from the dome 
of the diaphragm to the pubis) were imaged in the portal venous phase of enhancement (70-s delay), according to the protocol in our institution.

\subsection{Texture Analysis}

All images were analyzed by two radiologists ( 20 years and 5 years of abdominal imaging experience, respectively) who were blinded to the clinical outcome of the participants. Measurements were made on metastatic liver lesions according to the protocol provided by previous study [10], and tumor dimension and attenuation were measured using an image viewing software (Centricity, GE Medical Systems, Milwaukee, WI, USA). Texture analysis was carried out on the lesions using a commercial software (TexRAD, TexRAD Ltd., Cambridge, UK) by drawing a region of interest (ROI) around the peripheral margin of the lesions on CT images showing the largest area of the metastatic lesions. Various texture parameters were quantified prior and post to the application of spatial band-pass filters. Filter values on a scale of 0 to 6 were adjusted to select an appropriate scale. Where $0=$ absence of filtration (spatial scaling factor $(\mathrm{SSF})=0$ ), 2 = fine texture, 3 and 4 indicate different degrees of medium texture and 5 and 6 indicate different degrees of coarse texture. This analysis yielded derived CT texture images showing imaging characteristics at various spatial scales in the ROI (Figure 1).
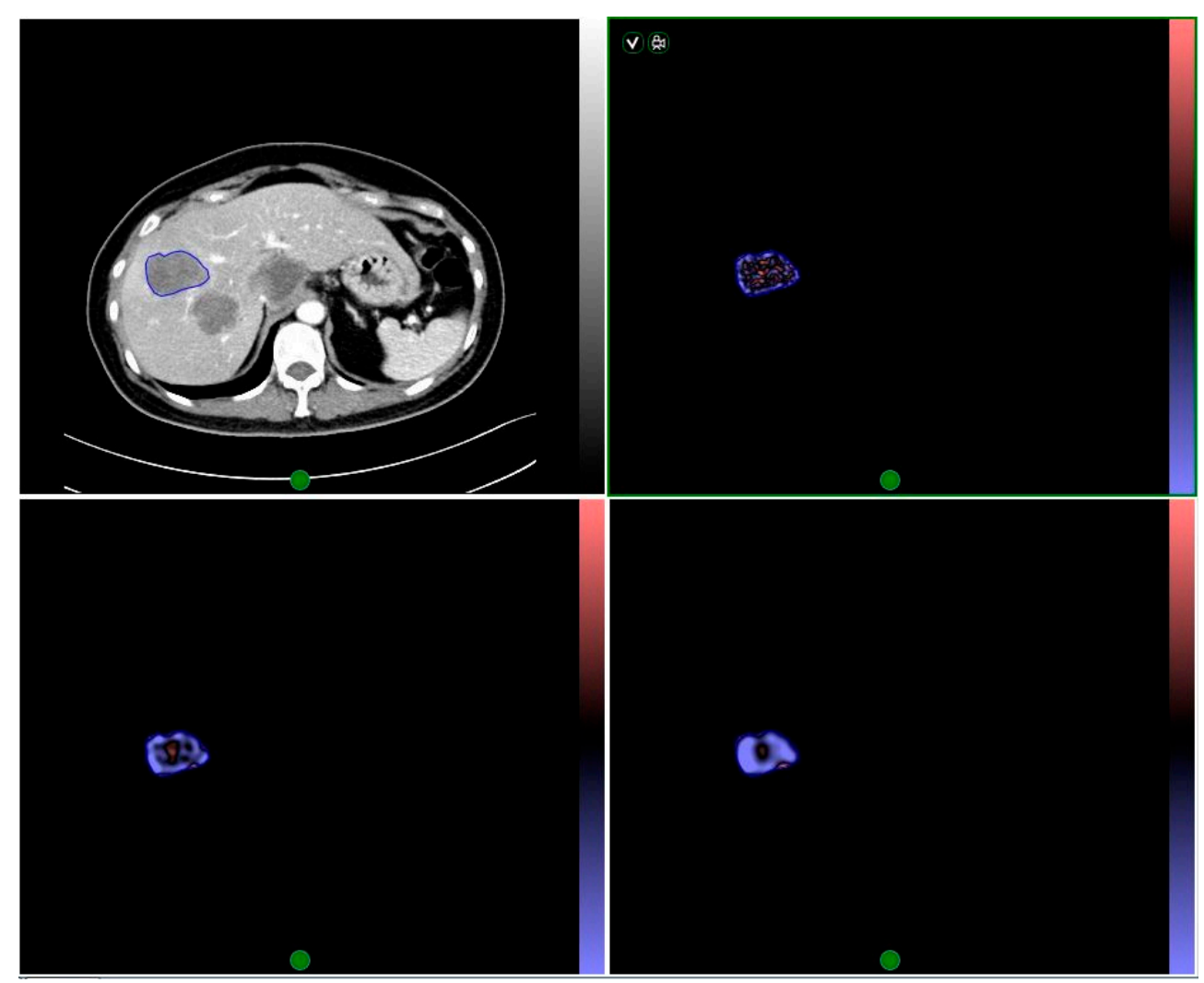

Figure 1. Illustration of lesion delineation, and image filtration at fine, medium, and coarse texture scales.

\subsection{Statistical Analysis}

Quantitative data were shown as mean \pm SD or medians. Categorical variables were presented as percentages. Mann-Whitney U test, Fisher exact, or Chi-square test were utilized appropriately to compare proportions and ranks of variables between training and validation cohorts. Texture parameters extracted from liver metastatic sites at baseline CT and at CT after two cycles of treatment, and the percentage change from the baseline value were all statistically analyzed. The association between texture parameters, RECIST 1.1, Choi, and modified Choi response criteria after two cycles of treatment and measured 
TTP were evaluated with the Kaplan-Meier analysis. Receiver operating characteristic (ROC) analysis was performed to identify threshold values for texture parameters. KaplanMeier analysis of participants with values below or above the thresholds were done to show the proportion of patients who did not have disease progression at any given time. Nonparametric log-rank test was performed to examine the differences between Kaplan-Meier curves for texture parameters, RECIST 1.1, Choi, and modified Choi criteria. Independent predictors of TTP among clinical and texture parameters were identified by performing multivariate analysis. All statistical analysis was done on SPSS 22.0 (IBM, New York, NY, USA). $p<0.05$ indicated a significant difference, however, when facing multiple comparisons, a stepwise Holm-Bonferroni procedure was performed to reduce the potential for type I errors [19].

\section{Results}

In this study, we finally analyzed 110 pancreatic cancer patients with liver metastasis. These patients were randomly divided into two cohorts with 60 participants (mean age of $61.1 \pm 8.6$ years; range, $42-76$ years) constituted a training cohort; while 50 participants (mean age, $56.1 \pm 10.3$ years; range, $31-73$ years) constituted the validation cohort. The demographics and clinical features did not show significant difference between the validation and training cohorts (Table 1$)$. In the training cohort, $10 \%$ of the patients $(6 / 60)$ exhibited more than three liver target lesions, while in the validation cohort, $24 \%$ of patients $(12 / 50)$ had >three target lesions. The initial treatment responses in the training cohort were shown in Table 2, categorized using RECIST 1.1, Choi, and modified Choi criteria. Follow-up imaging revealed that 42 of 60 patients in the training cohort and 32 of 50 patients in the validation cohort showed progression at the end of the follow-up. The median TTP was 178 (range, 36-1441) days in the validation cohort and 241 (range, 43-1164) days in the training cohort.

Table 1. Main baseline demographics and clinical characteristics of patients in the cohort.

\begin{tabular}{cccc}
\hline Characteristic & Training Cohort & Validation Cohort & $p$ Value \\
\hline Age $(\mathrm{y})$ & $61.1 \pm 8.6(42-76)^{1}$ & $56.1 \pm 10.3(31-73)^{1}$ & 0.055 \\
Gender & $36(60)$ & $28(56)$ & 0.765 \\
Male & $24(40)$ & $22(44)$ & \\
Female & $647.0(3430.2)\{1.2$ to & $778.4(1589.6)\{0.6$ to & 0.14 \\
CA 19-9 $(\mathrm{U} / \mathrm{mL})$ & $103,641.0\}^{2}$ & $23,661.0\}^{2}$ & 0.959 \\
ECOG Performance Status & $38(63)$ & $32(64)$ & \\
0 & $22(37)$ & $18(36)$ & 0.998 \\
1 & $22.2 \pm 2.5(17.3-28.7)^{1}$ & $22.3 \pm 3.4(18.6-35.5)^{1}$ & 0.707 \\
BMI $\left(\mathrm{kg} / \mathrm{m}^{2}\right)$ & $40(67)$ & $30(60)$ & \\
1 & $10(17)$ & $6(12)$ & \\
2 & $4(7)$ & $6(12)$ & \\
3 & $4(7)$ & $6(12)$ & 0.27 \\
4 & $2(3)$ & $178(206)\{36 \text { to } 1441\}^{2}$ & \\
$\geq 5$ & $241(260)\{43 \text { to } 1164\}^{2}$ & & \\
Number of target lesions & & & \\
Median time to progression & & & \\
\hline
\end{tabular}

Note. Unless otherwise indicated, data are number of patients, with percentages in parentheses. Bold means that the $p$ value lower than 0.05 is statistically significant. ${ }^{1}$ Data are mean \pm standard deviation, with range in parentheses for normally distributed data. ${ }^{2}$ Data are median with interquartile range in parentheses and minimum and maximum in braces for skewed data. 
Table 2. Response categorization of patients after two treatment cycles.

\begin{tabular}{cccc}
\hline Response Criteria & Partial Response & Stable Disease & Progressive Disease \\
\hline RECIST 1.1 & $16(27)$ & $36(60)$ & $8(13)$ \\
Choi & $42(70)$ & $6(10)$ & $12(20)$ \\
Modified Choi & $8(13)$ & $40(67)$ & $12(20)$ \\
\hline
\end{tabular}

Note. Data are numbers of patients. Data in parentheses are percentages.

The median values with their ranges for texture parameters for absolute scale values at baseline and after two treatment cycles, as well as corresponding percentage change, were shown in Table 3.

Table 3. Median value and percentage change for texture parameters for spatial scaling factor (SSF) at baseline and after two treatment cycles.

\begin{tabular}{|c|c|c|c|c|c|c|c|}
\hline & & $\mathrm{SSF}=0$ & $\mathrm{SSF}=2$ & $\mathrm{SSF}=3$ & $\mathrm{SSF}=4$ & $\mathrm{SSF}=5$ & $\mathrm{SSF}=6$ \\
\hline \multirow{3}{*}{ Entropy } & Baseline & 3.87 (3.01 to 4.45$)$ & 4.57 (3.68 to 5.18$)$ & 4.61 (3.78 to 5.37$)$ & 4.64 (3.38 to 5.54$)$ & 4.77 (2.99 to 5.73$)$ & 4.85 (2.62 to 5.89$)$ \\
\hline & $\begin{array}{c}\text { After Two } \\
\text { Treatment } \\
\text { Cycles }\end{array}$ & $3.94(2.46$ to 4.36$)$ & $4.62(2.56$ to 5.18$)$ & $4.59(2.56$ to 5.37$)$ & 4.69 (2.56 to 5.49$)$ & $4.72(2.56$ to 5.60$)$ & 4.73 (2.56 to 5.70$)$ \\
\hline & $\begin{array}{l}\text { Change } \\
\qquad \%)\end{array}$ & $\begin{array}{c}1.54(-32.60 \text { to } \\
16.57)\end{array}$ & $\begin{array}{c}0.29(-32.98 \text { to } \\
11.96)\end{array}$ & $\begin{array}{c}-0.15(-34.02 \text { to } \\
20.11)\end{array}$ & $\begin{array}{c}-0.49(-34.86 \text { to } \\
39.35)\end{array}$ & $\begin{array}{c}-1.22(-34.86 \text { to } \\
56.86)\end{array}$ & $\begin{array}{c}-1.53(-34.02 \text { to } \\
73.28)\end{array}$ \\
\hline \multirow{3}{*}{$\begin{array}{l}\text { Mean } \\
\text { intensity }\end{array}$} & Baseline & $\begin{array}{c}51.33(-0.34 \text { to } \\
95.51)\end{array}$ & $\begin{array}{c}-8.11(-71.55 \text { to } \\
1438.28)\end{array}$ & $\begin{array}{c}-18.05(-99.29 \\
\text { to } 2450.36)\end{array}$ & $\begin{array}{c}-21.23(-109.29 \\
\text { to } 2869.70)\end{array}$ & $\begin{array}{c}-23.40(-114.75 \\
\text { to } 2762.44)\end{array}$ & $\begin{array}{c}-17.35(-176.72 \\
\text { to } 2416.81)\end{array}$ \\
\hline & $\begin{array}{c}\text { After Two } \\
\text { Treatment } \\
\text { Cycles }\end{array}$ & $\begin{array}{c}51.45(-33.42 \text { to } \\
96.56)\end{array}$ & $\begin{array}{c}-7.94(-60.70 \text { to } \\
1575.50)\end{array}$ & $\begin{array}{c}-10.50(-82.04 \\
\text { to } 2823.12)\end{array}$ & $\begin{array}{c}-13.19(-110.35 \\
\text { to } 3050.04)\end{array}$ & $\begin{array}{c}-16.25(-120.03 \\
\text { to } 2680.73)\end{array}$ & $\begin{array}{c}-20.46(-105.19 \\
\text { to } 2166.58)\end{array}$ \\
\hline & $\begin{array}{l}\text { Change } \\
\qquad \%)\end{array}$ & $\begin{array}{c}2.01(-72.51 \text { to } \\
9729.41)\end{array}$ & $\begin{array}{c}22.94(-487.14 \text { to } \\
8807.41)\end{array}$ & $\begin{array}{c}-26.59(-924.15 \\
\text { to } 2780.51)\end{array}$ & $\begin{array}{c}-23.37(-642.47 \\
\text { to } 873.82)\end{array}$ & $\begin{array}{c}-16.46(-708.74 \\
\quad \text { to } 894.86)\end{array}$ & $\begin{array}{c}-22.06 \\
(-2135.06 \text { to } \\
1851.01)\end{array}$ \\
\hline \multirow{3}{*}{$\begin{array}{l}\text { Standard } \\
\text { devia- } \\
\text { tion }\end{array}$} & Baseline & $\begin{array}{c}15.00(6.78 \text { to } \\
27.15)\end{array}$ & $\begin{array}{c}36.56(19.36 \text { to } \\
211.51)\end{array}$ & $\begin{array}{c}40.15(13.39 \text { to } \\
339.41)\end{array}$ & $\begin{array}{c}45.44 \text { (9.40 to } \\
439.59)\end{array}$ & $\begin{array}{c}52.37(6.01 \text { to } \\
413.91)\end{array}$ & $\begin{array}{c}69.27(3.74 \text { to } \\
369.51)\end{array}$ \\
\hline & $\begin{array}{c}\text { After Two } \\
\text { Treatment } \\
\text { Cycles }\end{array}$ & $\begin{array}{c}15.39 \text { (8.59 to } \\
24.02)\end{array}$ & $\begin{array}{c}39.37(22.12 \text { to } \\
234.82)\end{array}$ & $\begin{array}{c}46.44(11.08 \text { to } \\
363.82)\end{array}$ & $\begin{array}{c}48.44(12.68 \text { to } \\
425.43)\end{array}$ & $\begin{array}{c}51.81(15.86 \text { to } \\
424.24)\end{array}$ & $\begin{array}{c}52.95(16.29 \text { to } \\
376.70)\end{array}$ \\
\hline & $\begin{array}{l}\text { Change } \\
(\%)\end{array}$ & $\begin{array}{c}5.02(-66.48 \text { to } \\
72.32)\end{array}$ & $\begin{array}{c}4.36(-61.88 \text { to } \\
98.55)\end{array}$ & $\begin{array}{c}-0.38(-70.23 \text { to } \\
248.47)\end{array}$ & $\begin{array}{c}-6.54(-80.10 \text { to } \\
436.38)\end{array}$ & $\begin{array}{c}-4.80(-82.34 \text { to } \\
662.06)\end{array}$ & $\begin{array}{c}-1.11(-82.07 \text { to } \\
917.91)\end{array}$ \\
\hline \multirow{3}{*}{ Skewness } & Baseline & $\begin{array}{c}0.14(-1.51 \text { to } \\
0.93)\end{array}$ & $\begin{array}{c}0.15(-2.78 \text { to } \\
3.81)\end{array}$ & $\begin{array}{c}0.18(-2.06 \text { to } \\
2.51)\end{array}$ & $\begin{array}{c}0.09(-2.23 \text { to } \\
4.67)\end{array}$ & $\begin{array}{c}0.13(-2.13 \text { to } \\
4.50)\end{array}$ & $\begin{array}{c}0.18(-1.61 \text { to } \\
3.91)\end{array}$ \\
\hline & $\begin{array}{c}\text { After Two } \\
\text { Treatment } \\
\text { Cycles }\end{array}$ & $\begin{array}{c}0.11(-0.71 \text { to } \\
1.14)\end{array}$ & $\begin{array}{c}0.27(-2.36 \text { to } \\
2.50)\end{array}$ & $\begin{array}{c}0.26(-1.07 \text { to } \\
3.50)\end{array}$ & $\begin{array}{c}0.11(-0.82 \text { to } \\
2.49)\end{array}$ & $\begin{array}{c}0.11(-1.36 \text { to } \\
1.78)\end{array}$ & $\begin{array}{c}0.31(-1.19 \text { to } \\
1.57)\end{array}$ \\
\hline & $\begin{array}{l}\text { Change } \\
(\%)\end{array}$ & $\begin{array}{c}-59.81(-550.00 \\
\quad \text { to } 250.00)\end{array}$ & $\begin{array}{c}-27.47(-766.67 \\
\text { to } 28200.00)\end{array}$ & $\begin{array}{c}-61.14 \\
(-2150.00 \text { to } \\
625.00)\end{array}$ & $\begin{array}{c}-62.57 \\
(-7300.00 \text { to } \\
2050.00)\end{array}$ & $\begin{array}{c}-21.86(-266.67 \\
\text { to } 3287.50)\end{array}$ & $\begin{array}{c}-22.59 \\
(-1433.33 \text { to } \\
622.22)\end{array}$ \\
\hline \multirow{3}{*}{ Kurtosis } & Baseline & $\begin{array}{c}-0.08(-1.32 \text { to } \\
4.32)\end{array}$ & $\begin{array}{c}0.12(-0.78 \text { to } \\
20.57)\end{array}$ & $\begin{array}{c}-0.17(-1.10 \text { to } \\
15.07)\end{array}$ & $\begin{array}{c}-0.46(-1.53 \text { to } \\
32.18)\end{array}$ & $\begin{array}{c}-0.63(-1.47 \text { to } \\
25.52)\end{array}$ & $\begin{array}{c}-0.59(-1.23 \text { to } \\
17.82)\end{array}$ \\
\hline & $\begin{array}{c}\text { After Two } \\
\text { Treatment } \\
\text { Cycles }\end{array}$ & $\begin{array}{c}-0.14(-1.06 \text { to } \\
1.67)\end{array}$ & $\begin{array}{c}-0.01(-0.95 \text { to } \\
16.10)\end{array}$ & $\begin{array}{c}-0.20(-1.22 \text { to } \\
17.78)\end{array}$ & $\begin{array}{c}-0.58(-1.61 \text { to } \\
6.91)\end{array}$ & $\begin{array}{c}-0.71(-1.47 \text { to } \\
6.66)\end{array}$ & $\begin{array}{c}-0.42(-2.23 \text { to } \\
3.41)\end{array}$ \\
\hline & $\begin{array}{l}\text { Change } \\
\qquad \%)\end{array}$ & $\begin{array}{c}-37.77(-723.53 \\
\text { to } 1566.67)\end{array}$ & $\begin{array}{c}-75.18(-700.00 \\
\quad \text { to } 2800.00)\end{array}$ & $\begin{array}{c}-95.27 \\
(-1133.33 \text { to } \\
466.24)\end{array}$ & $\begin{array}{c}-75.52 \\
(-6350.00 \text { to } \\
1081.25)\end{array}$ & $\begin{array}{c}-9.03(-937.11 \\
\text { to } 1257.14)\end{array}$ & $\begin{array}{c}-19.51(-700.00 \\
\text { to } 1566.67)\end{array}$ \\
\hline \multirow{3}{*}{$\begin{array}{l}\text { Mean of } \\
\text { positive } \\
\text { pixels }\end{array}$} & Baseline & $\begin{array}{c}51.46(23.50 \text { to } \\
95.51)\end{array}$ & $\begin{array}{c}24.51(8.95 \text { to } \\
1438.28)\end{array}$ & $\begin{array}{c}22.54(0.00 \text { to } \\
2450.36)\end{array}$ & $\begin{array}{c}25.58(0.00 \text { to } \\
2869.70)\end{array}$ & $\begin{array}{c}28.74(0.00 \text { to } \\
2762.44)\end{array}$ & $\begin{array}{c}34.58(0.00 \text { to } \\
2416.81)\end{array}$ \\
\hline & $\begin{array}{c}\text { After Two } \\
\text { Treatment } \\
\text { Cycles }\end{array}$ & $\begin{array}{c}51.50(0.00 \text { to } \\
96.56)\end{array}$ & $\begin{array}{c}26.14(15.61 \text { to } \\
1575.50)\end{array}$ & $\begin{array}{l}28.02(5.50 \text { to } \\
2823.12)\end{array}$ & $\begin{array}{c}31.03(6.50 \text { to } \\
3050.04)\end{array}$ & $\begin{array}{c}30.15(10.14 \text { to } \\
2680.73)\end{array}$ & $\begin{array}{c}37.87(4.59 \text { to } \\
2166.58)\end{array}$ \\
\hline & $\begin{array}{l}\text { Change } \\
\qquad \%)\end{array}$ & $\begin{array}{c}1.33(-100.00 \text { to } \\
68.28)\end{array}$ & $\begin{array}{c}6.97(-58.48 \text { to } \\
172.39)\end{array}$ & $\begin{array}{c}6.39(-64.31 \text { to } \\
2694.62)\end{array}$ & $\begin{array}{c}4.84(-73.93 \text { to } \\
4725.30)\end{array}$ & $\begin{array}{c}2.86(-84.20 \text { to } \\
3200.00)\end{array}$ & $\begin{array}{c}6.14(-82.33 \text { to } \\
7169.00)\end{array}$ \\
\hline
\end{tabular}


Predictive ability of texture parameters at different SSF were shown in Tables 4-6. With fine texture (SSF = 2), baseline MI and percentage change in both SD and kurtosis, were found to be significantly correlated with TTP. With medium texture $(\mathrm{SSF}=4)$, baseline kurtosis, and percentage change in MPP, were found to be significant predictors. Baseline kurtosis at SSF3, as well as percentage changes in entropy and MPP at SSF5, were also found to be significantly associated with TTP. Kaplan-Meier analysis of the proportion of patients without disease progression revealed significant differences for the above-mentioned texture parameters, which were better than those for RECIST 1.1, Choi-, and modified Choidefined responses after chemotherapy ( $p$ value $<0.05$ vs. $p$ value $=0.398, p$ value $=0.142$, and $p$ value $=0.536$, respectively) (Figure 2).

Table 4. ROC and Kaplan-Meier analysis for baseline texture parameters at different scale values.

\begin{tabular}{|c|c|c|c|c|c|c|c|}
\hline & & $\mathrm{SSF}=0$ & $\mathrm{SSF}=2$ & $\mathrm{SSF}=3$ & $\mathrm{SSF}=4$ & $\mathrm{SSF}=5$ & $\mathrm{SSF}=6$ \\
\hline \multirow{2}{*}{ Entropy } & ROC Threshold & $>3.52$ & $>4.52$ & $>4.64$ & $>4.88$ & $>4.93$ & $>4.89$ \\
\hline & $p$ Value & 0.55 & 0.60 & 0.20 & 0.28 & 0.27 & 0.10 \\
\hline \multirow{2}{*}{ Mean intensity } & ROC Threshold & $\leq 52.10$ & $>-9.49$ & $>-18.05$ & $>-19.54$ & $>34.04$ & $\leq-47.99$ \\
\hline & $p$ Value & 0.32 & 0.04 & 0.05 & 0.18 & 0.64 & 0.16 \\
\hline \multirow{2}{*}{$\begin{array}{l}\text { Standard } \\
\text { deviation }\end{array}$} & ROC Threshold & $>14.73$ & $>27.58$ & $>58.87$ & $>78.42$ & $>37.47$ & $>39.07$ \\
\hline & $p$ Value & 0.57 & 0.73 & 0.15 & 0.35 & 0.59 & 0.49 \\
\hline \multirow{2}{*}{ Skewness } & ROC Threshold & $>0.32$ & $>0.15$ & $>0.40$ & $>0.45$ & $\leq 0.03$ & $\leq 0.37$ \\
\hline & $p$ Value & 0.61 & 0.43 & 0.72 & 0.05 & 0.22 & 0.76 \\
\hline \multirow{2}{*}{ Kurtosis } & ROC Threshold & $>-0.06$ & $>0.01$ & $>0.10$ & $>-0.14$ & $\leq-0.97$ & $\leq-0.95$ \\
\hline & $p$ Value & 0.61 & 0.99 & 0.03 & 0.04 & 0.23 & 0.13 \\
\hline \multirow{2}{*}{$\begin{array}{l}\text { Mean of positive } \\
\text { pixels }\end{array}$} & ROC Threshold & $\leq 52.54$ & $>27.09$ & $>29.90$ & $>21.10$ & $>16.68$ & $>21.06$ \\
\hline & $p$ Value & 0.32 & 0.48 & 0.68 & 0.24 & 0.09 & 0.06 \\
\hline
\end{tabular}

Note. $p$ values were obtained with Kaplan-Meier analysis.

Table 5. ROC and Kaplan-Meier analysis for texture parameters after two treatment cycles at different scale values.

\begin{tabular}{cccccccc}
\hline & & SSF $=0$ & SSF $=2$ & SSF $=3$ & SSF $=4$ & SSF $=5$ & SSF $=\mathbf{6}$ \\
\hline \multirow{2}{*}{ Entropy } & ROC Threshold & $>4.13$ & $>4.93$ & $>4.63$ & $>4.92$ & $>4.97$ & $>4.99$ \\
& $p$ Value & 0.84 & 0.11 & 0.66 & 0.49 & 0.92 & 0.92 \\
\hline \multirow{2}{*}{ Mean intensity } & ROC Threshold & $\leq 54.58$ & $>-14.82$ & $>-10.50$ & $>-12.75$ & $>-38.76$ & $>-35.90$ \\
& $p$ Value & 0.06 & 0.59 & 0.08 & 0.06 & 0.78 & 0.25 \\
\hline Standard & ROC Threshold & $\leq 14.09$ & $>39.37$ & $>49.97$ & $>67.01$ & $>33.73$ & $>39.83$ \\
deviation & $p$ Value & 0.10 & 0.78 & 0.84 & 0.38 & 0.70 & 0.65 \\
\hline \multirow{2}{*}{ Skewness } & ROC Threshold & $\leq 0.03$ & $\leq 0.44$ & $\leq-0.17$ & $\leq-0.13$ & $>-0.46$ & $>-0.47$ \\
& $p$ Value & 0.79 & 0.56 & 0.06 & 0.11 & 0.70 & 0.30 \\
\hline \multirow{2}{*}{ Kurtosis } & ROC Threshold & $>-0.35$ & $>0.64$ & $>-0.29$ & $\leq-0.59$ & $\leq-0.80$ & $>-0.38$ \\
& $p$ Value & 0.62 & 0.21 & 0.53 & 0.62 & 0.36 & 0.22 \\
\hline Mean of positive & ROC Threshold & $\leq 54.58$ & $>25.63$ & $>104.25$ & $>33.97$ & $>30.15$ & $>12.15$ \\
pixels & $p$ Value & 0.06 & 0.31 & 0.50 & 0.47 & 0.33 & 0.70 \\
\hline Note $p$ valu
\end{tabular}

Note. $p$ values were obtained with Kaplan-Meier analysis.

After the performance of multivariate Cox regression analysis, baseline $\mathrm{MI}$ at fine texture scale $(\mathrm{SSF} 2, p$ value $=0.028)$, baseline kurtosis at medium texture scales (SSF3, $p$ value $=0.032 ;$ SSF4, $p$ value $=0.049)$, percentage change in kurtosis and SD at fine texture scale (SSF2, $p$ value $=0.033$ and $p$ value $=0.016$, respectively), and percentage change in MPP at medium texture scale (SSF4, $p$ value $=0.016$ ) were found to be independent prognostic factors for TTP (Table 7). 
Table 6. ROC and Kaplan-Meier analysis for percentage change in texture parameters at different scale values.

\begin{tabular}{cccccccc}
\hline & & SSF = & SSF = 2 & SSF = 3 & SSF = 4 & SSF = 5 & SSF = 6 \\
\hline \multirow{2}{*}{ Entropy } & ROC Threshold & $\leq 4.53$ & $\leq 0.66$ & $\leq 0.97$ & $\leq 3.34$ & $\leq 0.51$ & $\leq 8.85$ \\
& $p$ Value & 0.07 & 0.08 & 0.09 & 0.36 & $\mathbf{0 . 0 3}$ & 0.48 \\
\hline \multirow{2}{*}{ Mean intensity } & ROC Threshold & $>0.19$ & $\leq-119.23$ & $>-8.32$ & $>-73.13$ & $>-91.35$ & $>-82.74$ \\
& $p$ Value & 0.85 & 0.06 & 0.33 & 0.44 & 0.87 & 0.55 \\
\hline Standard & ROC Threshold & $\leq 48.66$ & $\leq-23.61$ & $\leq 75.13$ & $\leq 4.30$ & $\leq 8.05$ & $\leq 31.01$ \\
deviation & $p$ Value & 0.16 & $\mathbf{0 . 0 2}$ & 0.34 & 0.26 & 0.22 & 0.28 \\
\hline \multirow{2}{*}{ Skewness } & ROC Threshold & $>-59.81$ & $\leq 35.98$ & $\leq 94.54$ & $>-87.63$ & $>-37.60$ & $\leq-679.84$ \\
& $p$ Value & 0.26 & 0.54 & 0.34 & 0.17 & 0.62 & 0.95 \\
\hline \multirow{2}{*}{ Kurtosis } & ROC Threshold & $>-97.41$ & $>-112.04$ & $>-2.98$ & $>13.24$ & $>-32.10$ & $\leq-95.41$ \\
& $p$ Value & 0.19 & $\mathbf{0 . 0 2}$ & 0.89 & 0.33 & 0.73 & 0.86 \\
\hline Mean of positive & ROC Threshold & $>0.19$ & $\leq 6.65$ & $\leq 3.85$ & $\leq 2.62$ & $\leq 2.86$ & $\leq 12.84$ \\
pixels & $p$ Value & 0.80 & 0.10 & 0.67 & $\mathbf{0 . 0 2}$ & $\mathbf{0 . 0 0 5}$ & 0.22 \\
\hline Note $p$ values were obtained with Kaplan-Meier analysis & & & \\
\hline
\end{tabular}

Note. $p$ values were obtained with Kaplan-Meier analysis.
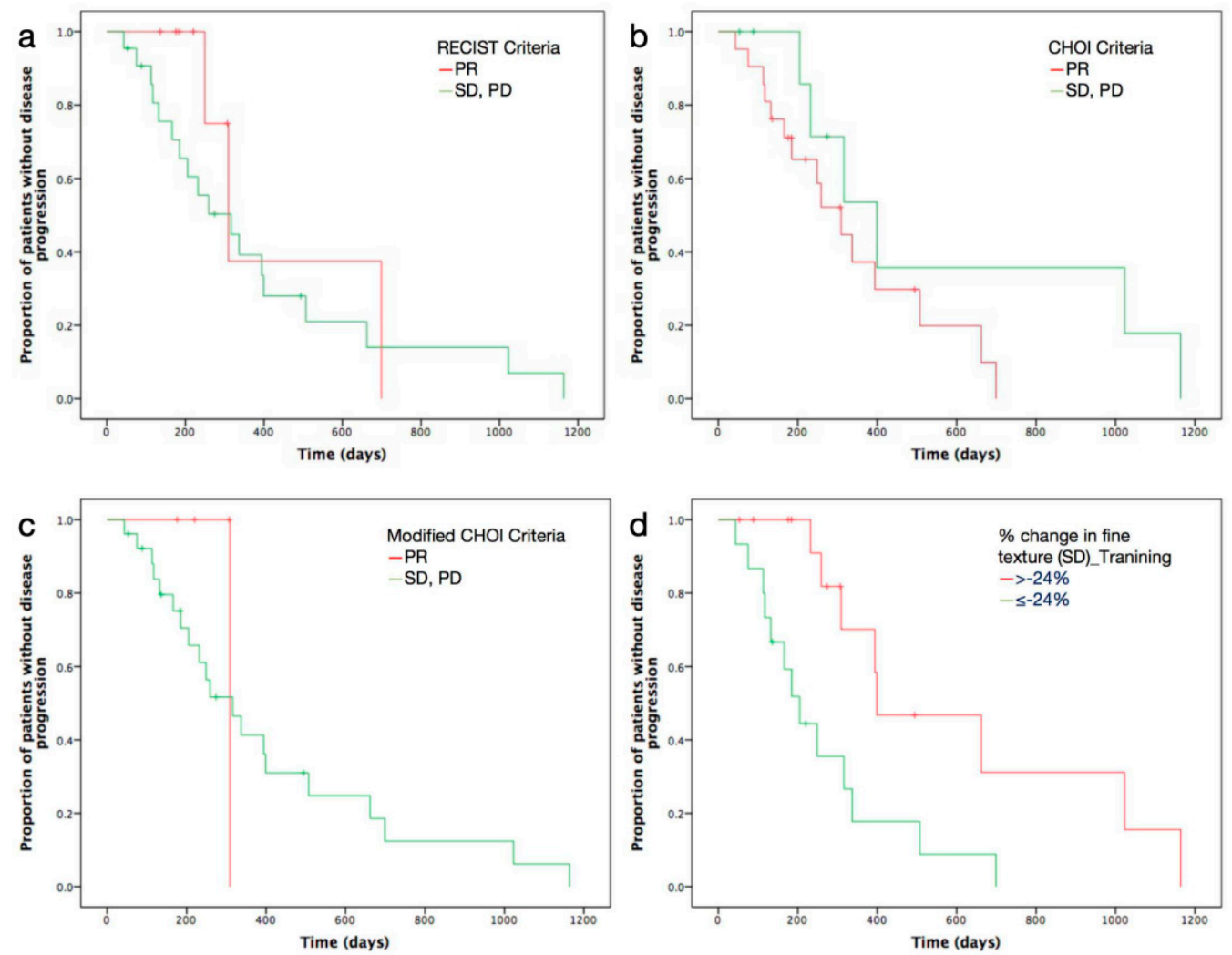

Figure 2. Kaplan-Meier curves show proportion of patients without disease progression for RECIST 1.1 (a), Choi (b), and modified Choi criteria (c) and percentage change in standard deviation (SD) (d). 
Table 7. Analysis of survival with multivariate cox regression model.

\begin{tabular}{|c|c|c|}
\hline \multirow{2}{*}{ Parameter } & \multicolumn{2}{|c|}{ Multivariate } \\
\hline & HR & $p$ Value \\
\hline \multicolumn{3}{|l|}{ Baseline (SSF = 2) } \\
\hline Age & $1.00(0.93,1.07)$ & 1.00 \\
\hline Gender & $1.13(0.43,2.96)$ & 0.80 \\
\hline CA19-9 & $0.20(0.02,1.63)$ & 0.13 \\
\hline BMI & $0.99(0.73,1.33)$ & 0.94 \\
\hline Mean intensity & $0.10(0.01,0.78)$ & 0.028 \\
\hline \multicolumn{3}{|l|}{ Baseline (SSF = 3) } \\
\hline Age & $0.99(0.93,1.06)$ & 0.84 \\
\hline Gender & $0.85(0.32,2.26)$ & 0.74 \\
\hline CA19-9 & $1.26(0.41,3.85)$ & 0.69 \\
\hline BMI & $1.06(0.83,1.34)$ & 0.66 \\
\hline Kurtosis & $0.33(0.12,0.91)$ & 0.032 \\
\hline \multicolumn{3}{|l|}{ Baseline $(\mathrm{SSF}=4)$} \\
\hline Age & $0.99(0.93,1.06)$ & 0.85 \\
\hline Gender & $0.73(0.27,1.95)$ & 0.53 \\
\hline CA19-9 & $1.24(0.39,3.88)$ & 0.72 \\
\hline BMI & $1.02(0.79,1.31)$ & 0.88 \\
\hline Kurtosis & $0.36(0.13,1.00)$ & 0.049 \\
\hline \multicolumn{3}{|l|}{ Percentage change $(\mathrm{SSF}=2)$} \\
\hline Age & $0.98(0.91,1.06)$ & 0.61 \\
\hline Gender & $1.80(0.59,5.46)$ & 0.30 \\
\hline CA19-9 & $1.12(0.36,3.51)$ & 0.85 \\
\hline BMI & $0.99(0.80,1.24)$ & 0.96 \\
\hline Standard deviation & $3.62(1.70,9.87)$ & 0.016 \\
\hline Kurtosis & $0.24(0.06,0.89)$ & 0.033 \\
\hline \multicolumn{3}{|l|}{ Percentage change $(\mathrm{SSF}=4)$} \\
\hline Age & $1.00(0.93,1.07)$ & 0.90 \\
\hline Gender & $0.70(0.26,1.88)$ & 0.48 \\
\hline CA19-9 & $1.68(0.56,5.07)$ & 0.36 \\
\hline BMI & $1.01(0.79,1.30)$ & 0.91 \\
\hline Mean of positive pixels & $3.45(1.27,9.41)$ & 0.016 \\
\hline \multicolumn{3}{|l|}{ Percentage change $(\mathrm{SSF}=5)$} \\
\hline Age & $1.00(0.93,1.07)$ & 0.89 \\
\hline Gender & $0.68(0.24,1.93)$ & 0.47 \\
\hline CA19-9 & $1.79(0.56,5.76)$ & 0.33 \\
\hline BMI & $1.02(0.80,1.30)$ & 0.88 \\
\hline Entropy & $0.99(0.21,4.69)$ & 0.99 \\
\hline Mean of positive pixels & $4.55(0.97,21.48)$ & 0.06 \\
\hline
\end{tabular}

Note. Data in parentheses are $95 \%$ confidence intervals. Bold means that the $p$ value lower than 0.05 is statistically significant.

However, these initially obtained results from training cohort needed further validation in an independent cohort. Optimal thresholds of above-mentioned independent texture parameters were acquired using ROC, and they were used to dichotomize texture parameters in the validation cohort. After application of Kaplan-Meier analysis, percentage change in SD at fine (SSF2) texture scale was confirmed as the only significant predictor of TTP ( $p$ value $=0.019)$ (Table 8$)$. Percentage change in SD exceeding $-24 \%$ at SSF2 was significantly associated with longer TTP following gemcitabine-based chemotherapy (Figure 3). 
Table 8. Univariate Kaplan-Meier analysis of texture parameters for predicting survival in the validation cohort.

\begin{tabular}{|c|c|c|c|c|}
\hline \multirow{2}{*}{ Parameter } & \multirow{2}{*}{ Value } & \multicolumn{2}{|c|}{ Median Time to Progression (d) } & \multirow{2}{*}{$p$ Value } \\
\hline & & Above Optimal Threshold & Below Optimal Threshold & \\
\hline \multicolumn{5}{|l|}{ Baseline } \\
\hline Mean intensity $(\mathrm{SSF}=2)$ & $-6.95[-53.76$ to 466.36$]$ & 179 (14) & $204(11)$ & 0.32 \\
\hline Kurtosis $(\mathrm{SSF}=3)$ & $0.12[-1.14$ to 29.18$]$ & 177 (15) & $242(10)$ & 0.37 \\
\hline Kurtosis $(\mathrm{SSF}=4)$ & $0.31[-1.15$ to 30.21$]$ & $204(14)$ & $175(11)$ & 0.29 \\
\hline \multicolumn{5}{|l|}{ Percentage change (\%) } \\
\hline Entropy $(\mathrm{SSF}=5)$ & $0.10[-16.80$ to 19.08$]$ & $177(10)$ & $204(15)$ & 0.97 \\
\hline Standard deviation $(\mathrm{SSF}=2)$ & $-0.51[-76.00$ to 72.79$]$ & $242(17)$ & $59(8)$ & 0.019 \\
\hline Kurtosis $(\mathrm{SSF}=2)$ & $-72.50[-2320.00$ to 781.82$]$ & 177 (18) & $204(7)$ & 0.35 \\
\hline Mean of positive pixels $(S S F=4)$ & $-8.67[-65.93$ to 321.14$]$ & $179(9)$ & 204 (16) & 0.90 \\
\hline Mean of positive pixels $(\mathrm{SSF}=5)$ & $-8.82[-82.09$ to 310.41$]$ & $179(9)$ & $204(16)$ & 0.75 \\
\hline
\end{tabular}

Note. Data are median with minimum and maximum in brackets and numbers in parentheses are numbers of patients. Bold means that the $p$ value lower than 0.05 is statistically significant.

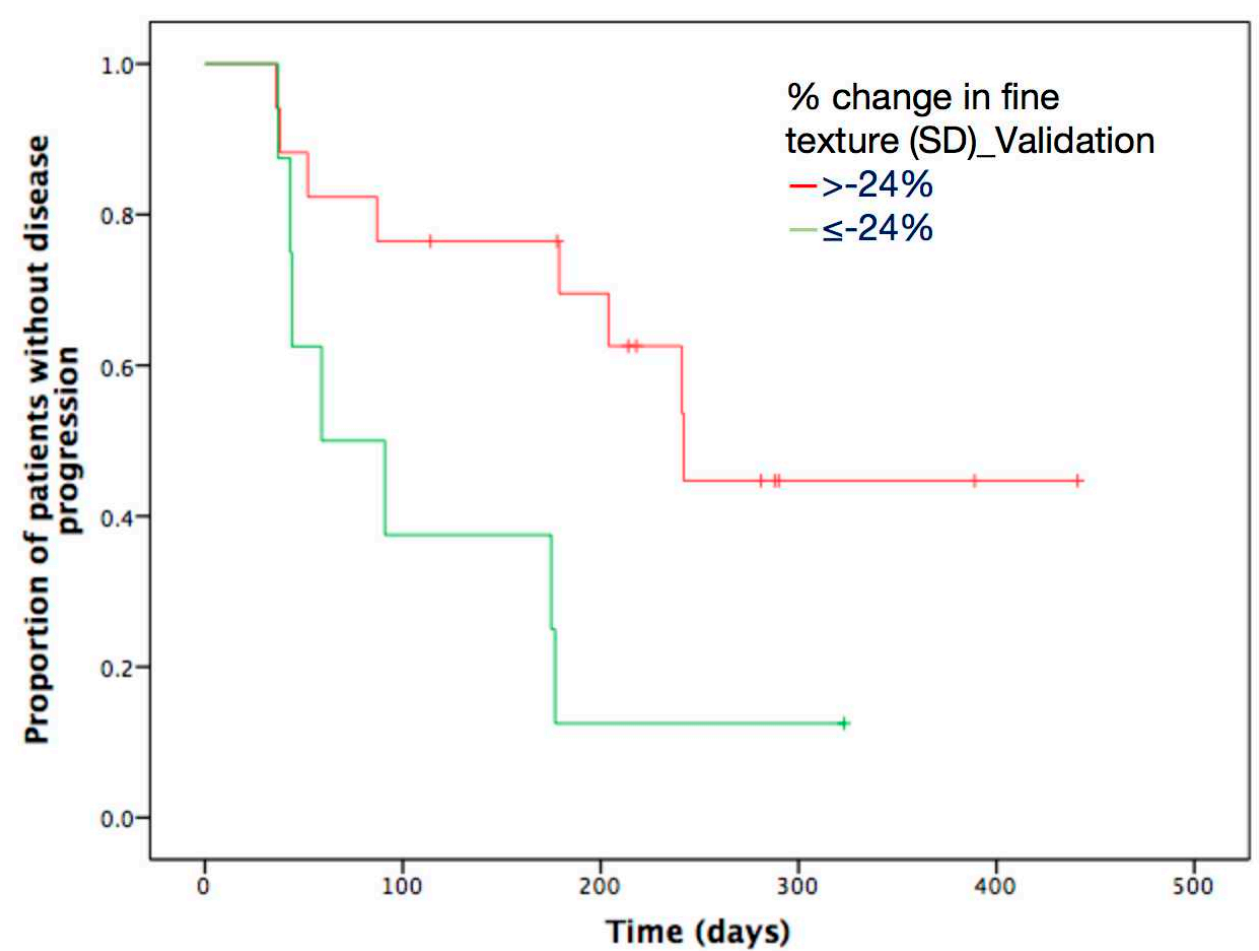

Figure 3. Kaplan-Meier survival curves according to percentage change in SD at fine (SSF2) texture scale in validation cohorts. Patients with percentage change in SD below optimal thresholds of -0.24 at fine texture scales showed significantly poorer survival in validation cohorts. TTP $=$ time to progression.

\section{Discussion}

In this study, we found that texture features of liver metastatic lesions on contrastenhanced CT images could accurately reflect treatment response and predict TTP in pancreatic cancer patients treated with chemotherapy, and these texture parameters showed great advantages over traditional assessment criteria. Importantly, we demonstrated that the percentage change in SD at fine texture scale had the ability to serve as an independent predictive biomarker, and its correlated optimal threshold value was validated in an independent cohort of patients.

RECIST 1.1 as a commonly used imaging-defined response assessment tool, has been widely used in the evaluation of various cancers. However, accurate assessment of clinical benefits from chemotherapy using RECIST 1.1 was constrained by the fact that many patients might exhibit tumor shrinkage to a degree that did not meet the criteria of RECIST 1.1, but still exhibited significant prolonged survival [10]. Tumor necrosis might 
occur in response to therapy without appreciable effects on tumor size, despite reduced tumor vascularization and tumor attenuation $[10,20,21]$. Therefore, response evaluation by RECIST 1.1 may significantly underestimate the number of patients experiencing treatment benefits [7]. Tumor attenuation was previously used to assess treatment response in other cancers [22]. Choi and modified Choi criteria, which included changes in tumor enhancement, were shown to be superior to RECIST 1.1 in their capacity to predict drug efficacy for various cancers $[8,9,23-28]$. Nevertheless, all these assessment tools did not reveal significant differences in Kaplan-Meier curves of the proportion of patients without disease progression, suggesting that existing assessment criteria were not sufficient to reflect underlying alterations in tumor heterogeneity. Therefore, efforts should be made to explore new assessment tools or biomarkers for the identification of pancreatic cancer patients with liver metastasis who might benefit from chemotherapy. Such biomarkers will inform the use of alternative therapies on possible non-responding patients earlier and help better understand disease heterogeneity.

Heterogeneity of neovascularization reflects conditions of hypoxia inside tumors, which is closely linked to high risk of invasion, metastasis, immunosuppression, and unfavorable response to chemotherapy [29]. Tumor response to a certain treatment could be obviously affected by its heterogenous blood supply. In this case, poor vascularization compromises drug availability at tumor sites. The emerging technique of CT texture analysis permits quantitative extraction of tumor heterogeneity, which enables assessment of tumor neoangiogenesis and hypoxia. Multiple previous studies have characterized the relationship between $\mathrm{CT}$ texture parameters and histopathological indicators of hypoxia or angiogenesis in various cancers $[30,31]$. CT texture analysis may also predict survival and response to treatment in cancer patients [10,32]. To our knowledge, the use of texture parameters as tumor response assessment criteria and predictive biomarkers in pancreatic cancer patients with liver metastasis undergoing chemotherapy has not been explored before.

Our results demonstrated the independent predictive significance of percentage change in liver metastatic lesion SD for TTP in pancreatic cancer patients with liver metastasis. The identified threshold values found by the training cohort were validated by another independent cohort, which increased the generalizability of the present findings [33]. SD is a measure of data dispersion from the mean and it increases in proportion to the square root of the number of objects highlighted and their mean intensity difference relative to background (i.e., dark and bright objects are both positive) [34]. Different from RECIST 1.1, Choi, and modified Choi criteria, the percentage change in texture SD (SSF $=2$ ) of liver metastatic lesions was an independent predictor of TTP. By applying a threshold of $-24 \%$ for SD at a fine scale (SSF $=2)$, the performance of the obtained Kaplan-Meier curves of proportion of patients without disease progression was notably different from and better than those obtained by RECIST 1.1, Choi, and modified Choi criteria. This revealed that $\mathrm{SD}$ as one of the indicators of tumor heterogeneity provided more details in the level of pixels or gray scales other than traditional enhancement and size change, thereby enabling complementary evaluation. This observation was supported by multiple recent studies involving unresectable pancreatic cancer and pancreatic head cancer [28]. Heterogeneity at fine texture scale was proposed to reflect lesion vascular permeability [35], which suggested that changes in vascular permeability caused by chemotherapy might explain the survival outcomes of pancreatic cancer patients with liver metastasis we observed in this study.

Due to its retrospective nature, this study was constrained by the limited number of participants, thus a prospective study with lager population needed to be conducted in the future to verify our conclusion. Therefore, it was still uncertain that CT texture of liver metastases could modify the clinical management of such patients, especially when multiple liver lesions or large, necrotic lesions presented. Ahn et al. [15] conducted a study to determine whether $\mathrm{CT}$ texture analysis of liver metastasis from colorectal cancer is predictive of therapeutic response after cytotoxic chemotherapy, and they found that the lower skewness and narrower SD showed good performance. Nakanishi et al. [36] 
also conducted a study focusing on the texture analsyis of liver metastasis, and they aimed to develop a radiomics-based prediction model for the response of colorectal liver metastases to oxaliplatin-based chemotherapy. Overall, 126 liver metastases were analyzed and multivariate analysis revealed that high radiomics scores was independently associated with good response. These above-mentioned studies might give us some inspiration for CT texture of liver metastases in response evaluation of pancreatic cancer. Another limitation was that only the largest cross-sectional area of the lesions, not the whole lesions, were analyzed, which therefore might not reflect the characteristics of liver metastatic lesions comprehensively. A 3D texture analysis software should be applied to evaluate the whole lesion heterogeneity and thus reproducibility of the study might be improved. Finally, selection bias could not be avoided as infiltrative metastatic lesions whose boundaries could not be clearly defined, were excluded from analysis.

\section{Conclusions}

Our findings indicated that the existing assessment criteria, including RECIST 1.1, Choi and modified Choi, were not sufficient for the evaluation of tumor response to treatment. We found that the percentage change in texture SD of liver metastatic lesions derived from contrast-enhanced CT texture analysis, might better predict tumor response and TTP in pancreatic patients with liver metastasis undergoing chemotherapy. Therefore, CT texture was proved to be an effective assessment tool and biomarker that predicted tumor response and TTP in a manner that was superior to traditional response criteria based on enhancement change, size change, or both.

Author Contributions: Conceptualization, S.C. and H.X.; methodology, S.C.; software, S.C.; validation, S.C.; formal analysis, S.C.; investigation, S.C.; resources, H.X.; data curation, S.C.; writingoriginal draft preparation, S.C.; writing-review and editing, H.X.; visualization, S.C.; supervision, H.X.; project administration, H.X.; funding acquisition, Z.J. All authors have read and agreed to the published version of the manuscript.

Funding: This research received no external funding.

Institutional Review Board Statement: Ethical review and approval were waived for this study, due to its retrospective design.

Informed Consent Statement: Patient consent was waived due to its retrospective design.

Data Availability Statement: The data presented in this study are available on request from the corresponding author. The data are not publicly available due to privacy.

Acknowledgments: The authors gratefully acknowledge Danqin Yi for her help in the manuscript preparation.

Conflicts of Interest: The authors declare no conflict of interest.

\section{References}

1. Siegel, R.L.; Miller, K.D.; Jemal, A. Cancer statistics, 2019. CA Cancer J. Clin. 2019, 69, 7-34. [CrossRef]

2. Kamisawa, T.; Wood, L.D.; Itoi, T.; Takaori, K. Pancreatic cancer. Lancet 2016, 388, 73-85. [CrossRef]

3. Hand, F.; Conlon, K.C. Pancreatic cancer. Surgery 2019, 37, 319-326. [CrossRef]

4. Sohal, D.P.; Mangu, P.B.; Khorana, A.; Shah, M.A.; Philip, P.A.; O’Reilly, E.M.; Uronis, H.E.; Ramanathan, R.K.; Crane, C.H.; Engebretson, A.; et al. Metastatic Pancreatic Cancer: American Society of Clinical Oncology Clinical Practice Guideline. J. Clin. Oncol. 2016, 34, 2784-2796. [CrossRef] [PubMed]

5. Conroy, T.; Desseigne, F.; Ychou, M.; Bouché, O.; Guimbaud, R.; Bécouarn, Y.; Adenis, A.; Raoul, J.-L.; Gourgou-Bourgade, S.; De La Fouchardière, C.; et al. FOLFIRINOX versus Gemcitabine for Metastatic Pancreatic Cancer. N. Engl. J. Med. 2011, 364, 1817-1825. [CrossRef] [PubMed]

6. Von Hoff, D.D.; Ervin, T.; Arena, F.P.; Chiorean, E.G.; Infante, J.; Moore, M.; Seay, T.; Tjulandin, S.A.; Ma, W.W.; Saleh, M.N.; et al Increased Survival in Pancreatic Cancer with nab-Paclitaxel plus Gemcitabine. N. Engl. J. Med. 2013, 369, 1691-1703. [CrossRef] [PubMed]

7. $\quad$ Eisenhauer, E.A.; Therasse, P.; Bogaerts, J.; Schwartz, L.H.; Sargent, D.; Ford, R.; Dancey, J.; Arbuck, S.; Gwyther, S.; Mooney, M.; et al. New response evaluation criteria in solid tumours: Revised RECIST guideline (version 1.1). Eur. J. Cancer 2009, 45, $228-247$. [CrossRef] [PubMed] 
8. $\quad$ van der Veldt, A.A.M.; Meijerink, M.R.; Eertwegh, A.J.M.V.D.; Haanen, J.B.A.G.; Boven, E. Choi response criteria for early prediction of clinical outcome in patients with metastatic renal cell cancer treated with sunitinib. Br. J. Cancer 2010, 102, 803-809. [CrossRef]

9. Nathan, P.; Vinayan, A.; Stott, D.; Juttla, J.; Goh, V. CT response assessment combining reduction in both size and arterial phase density correlates with time to progression in metastatic renal cancer patients treated with targeted therapies. Cancer Biol. Ther. 2010, 9, 15-19. [CrossRef] [PubMed]

10. Goh, V.; Ganeshan, B.; Nathan, P.; Juttla, J.K.; Vinayan, A.; Miles, K.A. Assessment of Response to Tyrosine Kinase Inhibitors in Metastatic Renal Cell Cancer: CT Texture as a Predictive Biomarker. Radiology 2011, 261, 165-171. [CrossRef]

11. Rao, S.-X.; Lambregts, D.; Schnerr, R.S.; Beckers, R.C.; Maas, M.; Albarello, F.; Riedl, R.G.; DeJong, C.H.; Martens, M.H.; Heijnen, L.A.; et al. CT texture analysis in colorectal liver metastases: A better way than size and volume measurements to assess response to chemotherapy? United Eur. Gastroenterol. J. 2016, 4, 257-263. [CrossRef] [PubMed]

12. Ganeshan, B.; Miles, K.A. Quantifying tumour heterogeneity with CT. Cancer Imaging 2013, 13, 140-149. [CrossRef] [PubMed]

13. Lubner, M.G.; Smith, A.D.; Sandrasegaran, K.; Sahani, D.V.; Pickhardt, P.J. CT Texture Analysis: Definitions, Applications, Biologic Correlates, and Challenges. Radiographics 2017, 37, 1483-1503. [CrossRef]

14. Cheng, S.-H.; Cheng, Y.-J.; Jin, Z.-Y.; Xue, H.-D. Unresectable pancreatic ductal adenocarcinoma: Role of CT quantitative imaging biomarkers for predicting outcomes of patients treated with chemotherapy. Eur. J. Radiol. 2019, 113, 188-197. [CrossRef] [PubMed]

15. Ahn, S.J.; Kim, J.H.; Park, S.J.; Han, J.K. Prediction of the therapeutic response after FOLFOX and FOLFIRI treatment for patients with liver metastasis from colorectal cancer using computerized CT texture analysis. Eur. J. Radiol. 2016, 85, 1867-1874. [CrossRef]

16. Beckers, R.C.J.; Trebeschi, S.; Maas, M.; Schnerr, R.S.; Sijmons, J.M.L.; Beets, G.L.; Houwers, J.B.; Beets-Tan, R.G.H.; Lambregts, D.M.J. CT texture analysis in colorectal liver metastases and the surrounding liver parenchyma and its potential as an imaging biomarker of disease aggressiveness, response and survival. Eur. J. Radiol. 2018, 102, 15-21. [CrossRef]

17. Borhani, A.A.; Dewan, R.; Furlan, A.; Seiser, N.; Zureikat, A.; Singhi, A.D.; Boone, B.; Bahary, N.; Hogg, M.E.; Lotze, M.; et al. Assessment of Response to Neoadjuvant Therapy Using CT Texture Analysis in Patients with Resectable and Borderline Resectable Pancreatic Ductal Adenocarcinoma. Am. J. Roentgenol. 2020, 214, 362-369. [CrossRef]

18. Tian, F.; Hayano, K.; Kambadakone, A.R.; Sahani, D.V. Response assessment to neoadjuvant therapy in soft tissue sarcomas: Using CT texture analysis in comparison to tumor size, density, and perfusion. Abdom. Imaging 2014, 40, 1705-1712. [CrossRef]

19. Aickin, M.; Gensler, H. Adjusting for multiple testing when reporting research results: The Bonferroni vs. Holm methods. Am. J. Public Health 1996, 86, 726-728. [CrossRef]

20. Joensuu, H.; Roberts, P.J.; Sarlomo-Rikala, M.; Andersson, L.C.; Tervahartiala, P.; Tuveson, D.; Silberman, S.L.; Capdeville, R.; Dimitrijevic, S.; Druker, B.; et al. Effect of the Tyrosine Kinase Inhibitor STI571 in a Patient with a Metastatic Gastrointestinal Stromal Tumor. N. Engl. J. Med. 2001, 344, 1052-1056. [CrossRef]

21. Faivre, S.; Sablin, M.-P.; Dreyer, C.; Raymond, E. Novel Anticancer Agents in Clinical Trials for Well-Differentiated Neuroendocrine Tumors. Endocrinol. Metab. Clin. N. Am. 2010, 39, 811-826. [CrossRef]

22. Tirkes, T.; Hollar, M.A.; Tann, M.; Kohli, M.D.; Akisik, F.; Sandrasegaran, K. Response Criteria in Oncologic Imaging: Review of Traditional and New Criteria. Radiographics 2013, 33, 1323-1341. [CrossRef] [PubMed]

23. Ronot, M.; Bouattour, M.; Wassermann, J.; Bruno, O.; Dreyer, C.; Larroque, B.; Castera, L.; Vilgrain, V.; Belghiti, J.; Raymond, E.; et al. Alternative Response Criteria (Choi, European Association for the Study of the Liver, and Modified Response Evaluation Criteria in Solid Tumors [RECIST]) Versus RECIST 1.1 in Patients with Advanced Hepatocellular Carcinoma Treated with Sorafenib. Oncologyst 2014, 19, 394-402. [CrossRef] [PubMed]

24. Luo, Y.; Chen, J.; Huang, K.; Lin, Y.; Chen, M.; Xu, L.; Li, Z.-P.; Feng, S.-T. Early evaluation of sunitinib for the treatment of advanced gastroenteropancreatic neuroendocrine neoplasms via CT imaging: RECIST 1.1 or Choi Criteria? BMC Cancer 2017, 17, 154. [CrossRef] [PubMed]

25. Choi, H.; Charnsangavej, C.; Faria, S.C.; Macapinlac, H.A.; Burgess, M.A.; Patel, S.R.; Chen, L.L.; Podoloff, D.A.; Benjamin, R.S. Correlation of Computed Tomography and Positron Emission Tomography in Patients with Metastatic Gastrointestinal Stromal Tumor Treated at a Single Institution with Imatinib Mesylate: Proposal of New Computed Tomography Response Criteria. J. Clin. Oncol. 2007, 25, 1753-1759. [CrossRef]

26. Benjamin, R.S.; Choi, H.; Macapinlac, H.A.; Burgess, M.A.; Patel, S.R.; Chen, L.L.; Podoloff, D.A.; Charnsangavej, C. We Should Desist Using RECIST, at Least in GIST. J. Clin. Oncol. 2007, 25, 1760-1764. [CrossRef]

27. Smith, A.D.; Lieber, M.L.; Shah, S.N. Assessing Tumor Response and Detecting Recurrence in Metastatic Renal Cell Carcinoma on Targeted Therapy: Importance of Size and Attenuation on Contrast-Enhanced CT. Am. J. Roentgenol. 2010, 194, 157-165. [CrossRef]

28. Smith, A.D.; Shah, S.N.; Rini, B.I.; Lieber, M.L.; Remer, E.M. Morphology, Attenuation, Size, and Structure (MASS) Criteria: Assessing Response and Predicting Clinical Outcome in Metastatic Renal Cell Carcinoma on Antiangiogenic Targeted Therapy. Am. J. Roentgenol. 2010, 194, 1470-1478. [CrossRef]

29. Yun, Z.; Lin, Q. Hypoxia and Regulation of Cancer Cell Stemness. In Tumor Microenvironment and Cellular Stress; Springer: Berlin/Heidelberg, Germany, 2014; pp. 41-53. [CrossRef]

30. Ganeshan, B.; Goh, V.; Mandeville, H.C.; Ng, Q.S.; Hoskin, P.J.; Miles, K.A. Non-Small Cell Lung Cancer: Histopathologic Correlates for Texture Parameters at CT. Radiology 2013, 266, 326-336. [CrossRef] 
31. Hayano, K.; Tian, F.; Kambadakone, A.R.; Yoon, S.S.; Duda, D.G.; Ganeshan, B.; Sahani, D.V. Texture Analysis of Non-ContrastEnhanced Computed Tomography for Assessing Angiogenesis and Survival of Soft Tissue Sarcoma. J. Comput. Assist. Tomogr. 2015, 39, 607-612. [CrossRef]

32. Durot, C.; Mulé, S.; Soyer, P.; Marchal, A.; Grange, F.; Hoeffel, C. Metastatic melanoma: Pretreatment contrast-enhanced CT texture parameters as predictive biomarkers of survival in patients treated with pembrolizumab. Eur. Radiol. 2019, 29, 3183-3191. [CrossRef] [PubMed]

33. Taylor, J.M.G.; Ankerst, D.; Andridge, R. Validation of Biomarker-Based Risk Prediction Models. Clin. Cancer Res. 2008, 14, 5977-5983. [CrossRef] [PubMed]

34. Miles, K.A.; Ganeshan, B.; Hayball, M.P. CT texture analysis using the filtration-histogram method: What do the measurements mean? Cancer Imaging 2013, 13, 400-406. [CrossRef] [PubMed]

35. Bezy-Wendling, J.; Kretowski, M.; Rolland, Y.; Le Bidon, W. Toward a better understanding of texture in vascular CT scan simulated images. IEEE Trans. Biomed. Eng. 2001, 48, 120-123. [CrossRef] [PubMed]

36. Nakanishi, R.; Oki, E.; Hasuda, H.; Sano, E.; Miyashita, Y.; Sakai, A.; Koga, N.; Kuriyama, N.; Nonaka, K.; Fujimoto, Y.; et al. Radiomics Texture Analysis for the Identification of Colorectal Liver Metastases Sensitive to First-Line Oxaliplatin-Based Chemotherapy. Ann. Surg. Oncol. 2021, 28, 2975-2985. [CrossRef] [PubMed] 